\title{
Experimental determination of the energy efficiency of rotors of vertical-axis wind turbines for autonomous power supply on land and at sea
}

\author{
Nikolai Shishkin ${ }^{1, *}$ and Roman Il'in ${ }^{1}$ \\ ${ }^{1}$ Laboratory of Non-conventional Energy of the Department of Energy Problems of the Saratov \\ Scientific Center of the RAS at Astrakhan State Technical University, 16, Tatischeva Str., 414025, \\ Russia
}

\begin{abstract}
For the first time, experimental studies have been performed to assess the effect of flaps with triangular elements on the operation of the NDarrieus and Savonius rotors. It has been established that the rotational speed of the original N-Darrieus and Savonius rotors having blades with flaps is greater than the rotation frequency of the N-Darrieus and Savonius rotors of the known construction by $18 \%$, and the power factors of the NDarrieus and Savonius rotors are for $17 \%$ and $19 \%$ more. Optimization of the geometrical parameters of the blades can lead to an increase in the power factor of the N-Darrieus rotor up to a value of 0.72 , which is higher than the maximum possible value of 0.45 for the horizontal axis wind turbine. It is advisable to aggregate N-Darrieus rotors with Savonius rotors having a large starting torque. The upgraded N-Darrieus and Savonius rotors, as well as the combined rotors based on them, can be used in power supply systems for various objects on land and at sea.
\end{abstract}

\section{Introduction}

In many countries, decentralized power supply systems using wind turbines (WT) are becoming increasingly common [1-8]. In addition to horizontal axial wind turbines (HO WT), vertical-axial wind turbines (VA WT) are now used. The advantages of all VA WTs is that the operation of these units does not depend on the wind direction. The most widely used are 2 types of VA WT based on the N-Darrieus rotor with vertical blades of the airfoil and Savonius rotor with vertical semi-cylindrical blades [9-23]. The advantages of the N-Darrieus rotors are their rapidity, and the disadvantage is the impossibility of self-starting due to the small starting torque. The advantages of the Savonius rotors are the possibility of their selfstarting due to the large starting torque, and the disadvantage is its low rotation speed. These advantages of VA WT rotors compared to the most widespread HO WTs make them an ideal source of low power for consumers of electrical and heat energy of low-rise buildings, farms, tourist, industrial and other facilities remote from centralized water supply, electricity, heating and gas supply systems. These rotors and their various combinations can be used not

\footnotetext{
*Corresponding author: n.shishkin-53@mail.ru, kaften.astu@mail.ru
} 
only on land, but also at sea to power the ships and to drive the propellers of small boats [24, 25].

However, as noted by most authors, the N-Darrieus rotor energy efficiency (power factor) does not exceed 0.35, and for Savonius rotor is not more than 0.20 , which is much less than the propeller-type wind wheel of the horizontal axis WTs [4, 8-16].

The rotation of the N-Darrieus rotors, as well as the HO WT rotors, according to most authors, is due to the lifting force arising in the wing profile of the blade, and the Savonius rotor rotates due to the difference in drag forces acting on the blades located on opposite sides of the rotor axis [9-16]. However, a number of authors [17-20] believe that lifting force occurs only on a small part of the circular trajectory of the wing blade, and on most of the trajectory the flow around the blade occurs in a pulsating flow, similar to that arising from the interaction of horizontal air flow with a flapping bird wing.

The authors of the article believe that to increase energy efficiency by reducing losses from large vortices formed at the ends of the blades of the wing profile of the N-Darrieus rotor, as well as at the ends of the semi-cylindrical blades of Savonius rotor, end elements of the blades (flaps) of various shapes similar to the wings of birds can be used. All wings have triangular, rounded or rectangular ends on the back [21]. Apparently, due to the long evolution, such wings appeared which allows, due to perfect aerodynamics, reducing the energy consumption per flight, both during lifting due to wing flaps and due to soaring in a counter-flow. The authors propose to extend this experience to similar technical objects, such as blades of the N-Darrieus and Savonius rotor.

As a result of studies of the "ideal N-Darrieus rotor", which has only blades and no traverse, creating additional resistance and vortex flows that impede the operation of the blades, $\mathrm{Cp}=0.72$ was obtained [17-19]. This is above the limit value $\mathrm{Cp}=0.59$ for an ideal HO WT rotor. However, these results require verification, since they were obtained in a hydraulic tray under conditions different from those observed under actual operating conditions of the HE WT. In wind tunnels and hydraulic trays in some cases there is an actual increase in the flow velocity in the cross section of the rotor, which can lead to a significant increase in power factor.

Many research works are devoted to the experimental study of the Savonius two-tier rotor $[16,19,22,26]$. Experimental studies carried out in a wind tunnel [26] showed that with an increase of the flow blocking coefficient $\sigma$ from $5 \%$ to $20 \%$, the power factor CP increases by a factor of 2 . In this case, the maximum $\mathrm{CP}$ is achieved at a higher specific velocity $\mathrm{Z}$. The author of [22] concludes that the Savonius optimal rotor should have the following values of its geometrical parameters: $\mathrm{H} / \mathrm{D}=0.77, \mathrm{p} / \mathrm{R}=0.20, \theta=135^{\circ}, \mathrm{D} / \mathrm{D} 1=0.75$. However, experiments carried out in the hydrobasin with practically no influence of the flow boundaries showed that for the Savonius rotor with dimensions $R=0.068 \mathrm{~m}, \mathrm{p}=0.015 \mathrm{~m}(\mathrm{p} / \mathrm{R}=0.22)$, $\mathrm{H}=0.175 \mathrm{~m}, \mathrm{D}=0.26 \mathrm{~m}(\mathrm{H} / \mathrm{D}=0.77)$ and $\theta=135^{\circ}$ [19], close to the recommended values [22], the maximum value of the power factor is achieved for the coefficient of rapidity $\mathrm{Z}=$ 0.65 and is $\mathrm{CP}=0.20$.

Thus, it seemed appropriate to test the N-Darrieus and Savonius rotors of optimal geometry in an unrestricted airflow. Such a flow can be simulated in the first approximation by the flow of air from a fan with a wheel of a sufficiently large diameter, 2-3 times larger than the diameter of the rotor. In addition, it is necessary to evaluate the effect of flaps in the form of triangular zigzags on the parameters of the Savonius rotor, in particular, on the shaft rotation frequency $\mathrm{n}$ and the power factor $\mathrm{CP}$.

The aim of the work is to develop advanced VA WTs based on experimental study of their energy efficiency. The main tasks of the work are: experimental research and analysis of energy efficiency of N-Darrieus and Savonius rotors when using blades having flaps with triangular elements, as well as developing recommendations for improving their designs to increase the energy efficiency of various types of rotors VA WT. 


\section{Experimental technique}

At the Laboratory of Non-conventional Energy of the Department of Energy Problems of the Saratov Scientific Center of the Russian Academy of Sciences at Astrakhan State Technical University, a laboratory setup was developed for an experimental study of the efficiency of VA WT rotors of various types. The experimental setup of the authors has a source of air flow fan, which directs the free stream of air that simulates the wind to the rotor. Therefore, the results obtained are fairly reliable for all N-Darrieus and Savonius rotors under consideration.

The experimental setup is shown in fig. 1 . The source of the air flow was fan 1 with speed switch 2. The coordinate device 3 in the form of a frame with a grid of thick synthetic filaments of $3 \times 3$ cells with dimensions of $0.10 \times 0.10 \mathrm{~m}$ was used to determine the air flow rate at 9 points (cell centers). The average air flow rate $V$ in each section at a distance $X$ from the fan was determined by averaging the flow velocities at 9 points using a digital anemometer 4 with an error not exceeding $0.1 \mathrm{~m} / \mathrm{s}$. By changing the distance to the rotor axis and switching speeds, the averaging over the cross section of the air flow speed was made. The rotational moment of the rotor was balanced by the moment of elastic force of the stretchable spring (dynamometer) 6. The left fixed part was attached to a synthetic thread, which passed through the pulley 5 and was attached to a fixed rod. The deformation of the spring was measured on a scale 7 . The rotational speed of the rotor was measured using a DC microelectric generator 10 with a V-belt multiplier 9 with a gear ratio of 7.09 for a corresponding increase in the rotation frequency of the generator shaft.

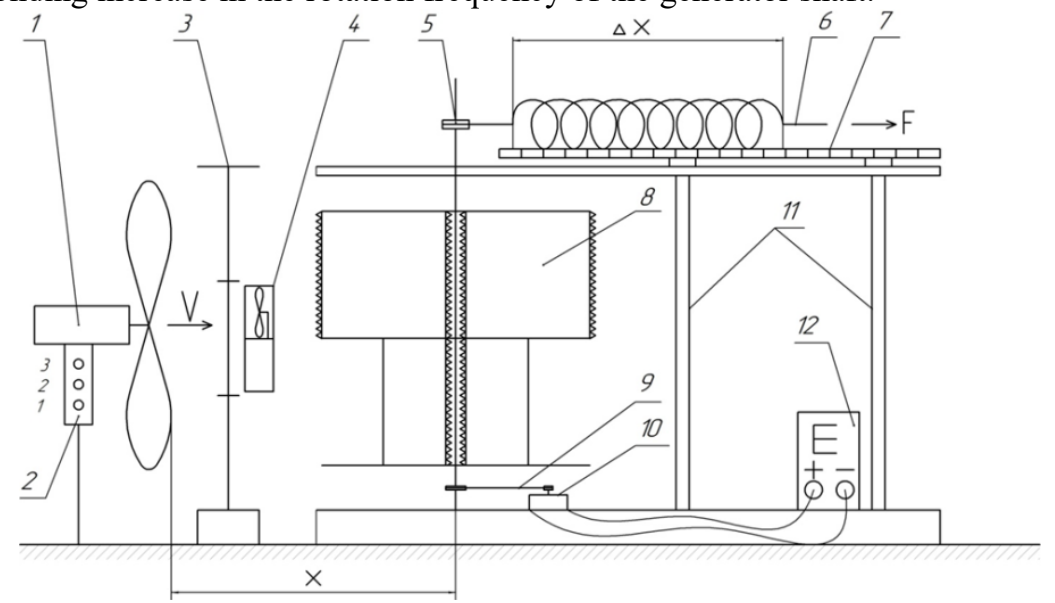

Fig. 1. The scheme of experimental setup for investigation rotors Savonius and N-Darrieus: 1 - fan; 2 - speed switch; 3 - grid 3x3; 4 - anemometer; 5 - pulley; 6 - spring; 7 - scale; 8 - Savonius rotor having blades with flaps; 9 - multiplier; 10 - motor drive; 11 - stands; 12 - voltmeter.

The laboratory setup was used for investigations the energy parameters of four types of rotor: Savonius rotor, Savonius rotor with blades having flaps, N-Darrieus rotor and NDarrieus rotor with blades having flaps. A general view of the experimental laboratory setup with Savonius and N-Darrieus rotors with flaps is shown in Figure 2. 


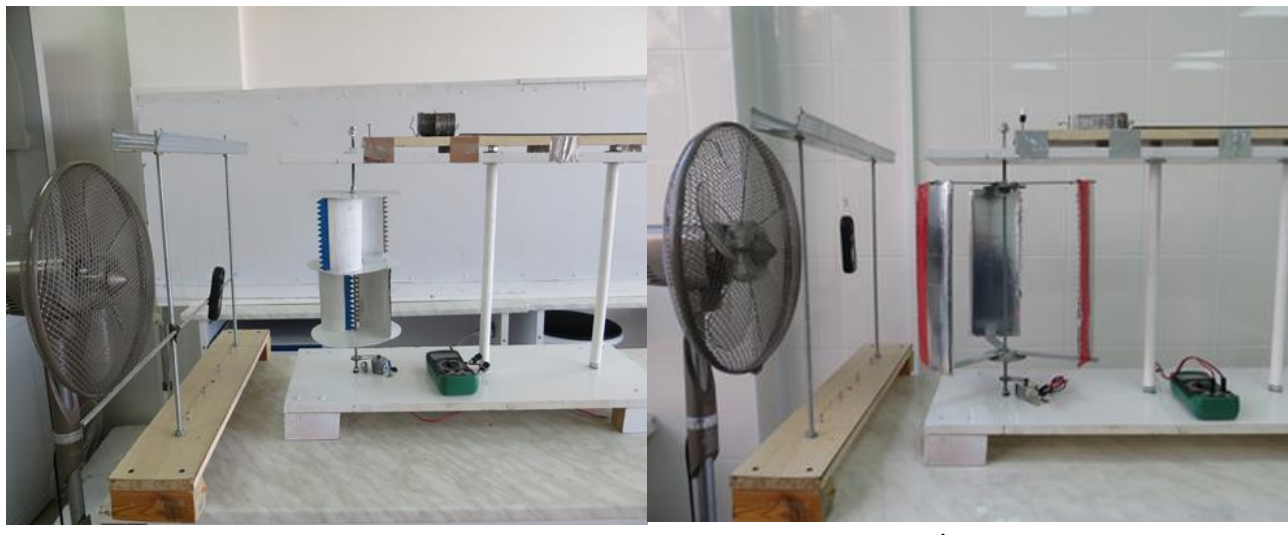

a

b

Fig. 2. General view of the experimental laboratory setup with Savonius and N-Darrieus rotors with flaps

Figure 2a shows a general view of the laboratory setup for studying Savonius rotor parameters of optimal geometry with a diameter of $D=0.185 \mathrm{~m}$, and 4 semi-cylindrical blades with a disclosure angle $\theta=135^{\circ}$ and diameter $D_{\mathrm{bl}}=0.100 \mathrm{~m}$. The blades' height $H=0.140 \mathrm{~m}$ and they are attached to the shaft with horizontal disks of diameter $D=0.246 \mathrm{~m}$. The blades have front and rear generators with triangular elements (flaps) with a height of $0.010 \mathrm{~m}$. Figure $2 \mathrm{~b}$ shows a general view of the laboratory setup with the N-Darrieus rotor with a diameter $\mathrm{D}_{\mathrm{D}}=0.300 \mathrm{~m}$ with three blades with profile width $b_{\mathrm{D}}=0.100 \mathrm{~m}$, height $H_{D}$ and thickness $\delta_{\mathrm{D}}=0.012 \mathrm{~m}$. The blades have flaps with triangular elements of $0.010 \mathrm{~m}$ height.

Processing of measurement results was carried out in the following sequence.

According to readings from a digital voltmeter, which measures the EMF of electric generator $E$, the rotational speed of the corresponding rotor was determined

$$
n=K_{n} E, \mathrm{rpm},
$$

where $K_{n}$ is calibration constant, rpm*V.

Useful power of rotor was determined according to the formula:

$$
P_{R}=M_{R} \omega=\frac{1}{60} K_{G} \Delta x D_{S} \pi n, \mathrm{n}^{*} \mathrm{~m}
$$

where $M_{R}$ is rotor torque balanced by the moment of elastic force of stretchable spring (dynamometer), $\mathrm{n}^{*} \mathrm{~m}$; $\omega$ is rotor angular frequency of, $\mathrm{s}^{-1} ; K_{G}$ is calibration coefficient of dynamometer, $\mathrm{n} / \mathrm{m} ; D_{S}$ is diameter of the pulley for measuring the elastic force, $\mathrm{m}$.

The specific velocity of rotors was determined by the known formula

$$
Z=\frac{\omega D_{R}}{2 \mathrm{~V}}=\frac{\pi n D_{R}}{60 \mathrm{~V}}
$$

where $V$ is velocity of the air flow (wind), $\mathrm{m} / \mathrm{s} ; n$ is rotor frequency, rpm.

Power factor was determined using the formula suitable for any wind turbine:

$$
C_{P}=\frac{P_{R}}{P}=\frac{2 P_{R}}{\rho S V^{3}},
$$

where $P$ is power of air flow, W; $S_{R}=D_{R} H_{R}$ is swept area, $\mathrm{m}^{2} ; \rho$ is air density, $\mathrm{kg} / \mathrm{m}^{3}$. According to the experimental results we created plots (Figure 3) showing relation between rotation frequency of the N-Darrieus and Savonius rotors $(n)$ and wind speed $(V)$, measured in the range from 2 to $6 \mathrm{~m} / \mathrm{s}$. 

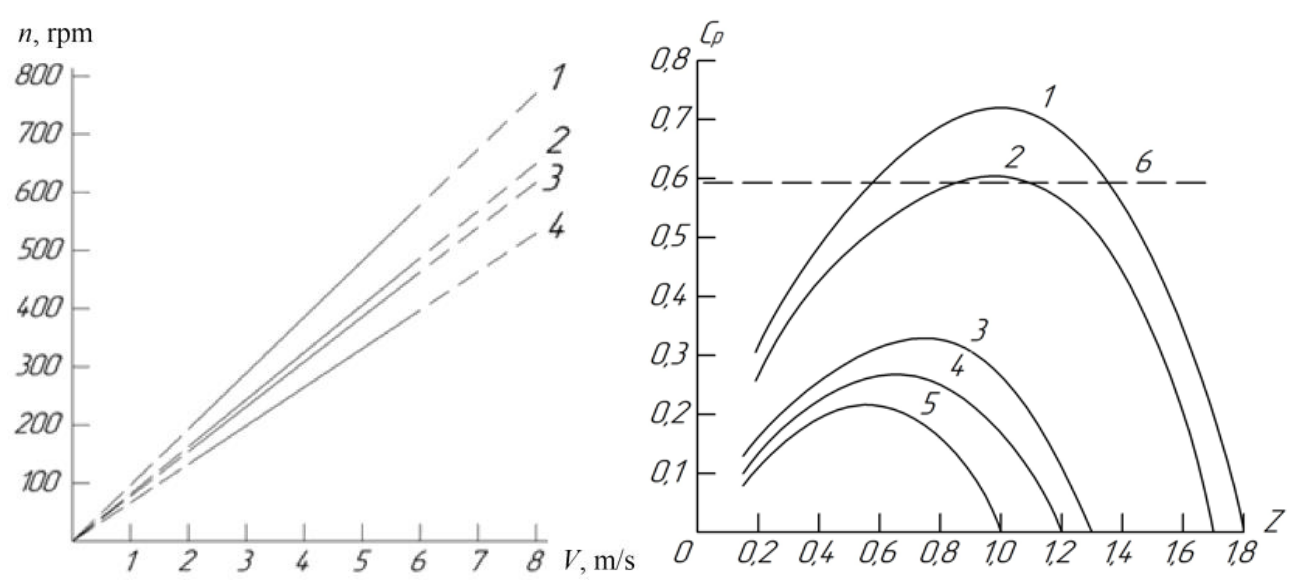

Fig. 3. Relation between rotor frequency for N- Fig. 4. Relation between power factor for NDarrieus and Savonius rotors $(n)$ and wind speed Darrieus and Savonius rotors $\left(C_{\mathrm{P}}\right)$ and specific $(V)$ velocity $(Z)$

Figure 4 shows relation between power factor for N-Darrieus and Savonius rotors $\left(C_{\mathrm{P}}\right)$ and specific velocity $(Z)$.

\section{Results and discussion}

As can be seen from Figure 3, the rotation frequency of the original N-Darrieus rotor having blades with flaps (line 1) is 18\% higher than the rotation frequency of the N-Darrieus rotor of known design (line 2). This is explained by the fact that flaps with triangular elements reduce the losses arising due to large vortices in the rear part of the blade, breaking them into a great number of small ones. This leads to an increase in the N-Darrieus rotor frequency. The frequency of rotation of the original Savonius rotor having blades with flaps (line 3) is $18 \%$ higher than the frequency of rotation of the Savonius rotor of known design (line 2). This is due to the fact that zigzag flaps increase the drag forces that are the driving force in the Savonius rotors, as well as reduce the losses arising from large vortices, breaking them into many small ones. All this helps to increase the rotors frequencies.

As it can be seen from Figure 4, all curves of rotor power factors $C_{P}$ have a pronounced maximum for different values of $Z$, depending on the rotor design. The obtained maximum values of power factor for the $\mathrm{N}$-Darrieus rotor are almost equal to that for the optimal design of the N-Darrieus rotor, but with a profile filling factor of $\sigma \approx 0.65$ with specific speed $Z=$ 1.1 is $C_{P}=0.60$, and for the N-Darrieus rotor having blades with flaps $C_{P}=0.70$. These values exceed $C_{P}=0.59$ for an ideal HO WT rotor (line 6), which is unattainable for actually operating units of this type. It is also seen that the power factor of the N-Darrieus rotor having blades with flaps (line 1) is $17 \%$ higher than the rotational speed of the N-Darrieus rotor of known design (line 2). This is because the flaps reduce the loss in the back of the blade. This leads to an increase in $C_{P}$ almost as much as the rotation frequency of the N-Darrieus rotor changes.

The power factor $C_{\mathrm{P}}$ of the original Savonius rotor having blades with flaps (line 3) is $19 \%$ more than power factor of the Savonius rotor of known design (line 4). This is due to the fact that zigzag flaps increase drag forces and reduce losses arising from large vortices. This leads to an increase in $C_{\mathrm{P}}$ almost as much as the rotational speed of the Savonius rotor increases. The maximum $C_{\mathrm{P}}$ values obtained by the authors for the Savonius rotor of optimal design and the original Savonius rotor with flaps on blades were $C_{\mathrm{P}}=0.27$ and $C_{\mathrm{P}}=0.32$ at specific velocities equal to $Z=0.70$ and $Z=0.80$, respectively. For comparison, Figure 4 
shows the dependence of $C_{P}=f(Z)$ (curve 6) obtained from the study of the Savonius rotor in a hydrobasin [19]. In this case, the maximum value of the power factor is achieved at specific velocity equal to $\mathrm{Z}=0.65$ and is $C_{P}=0.20$ (line 5 in Figure 4).

The obtained maximum values of power factor for the N-Darrieus rotor with practically optimal design, but with a profile filling factor $\sigma \approx 0.65$ and specific velocity $Z=1.1$ are $C_{\mathrm{P}}$ $=0.60$, and for the N-Darrieus rotor having blades with flaps this characteristics equals to $C_{\mathrm{P}}$ $=0.70$. These values exceed $C_{\mathrm{P}}=0.59$ (line 6 in Fig. 4) for an ideal HO WT rotor, which is unattainable for actually operating units of this type. The maximum $C_{\mathrm{P}}$ values for the Savonius rotor of optimal design and for original Savonius rotor with flaps on the blades were $C_{\mathrm{P}}=0.27$ and $C_{\mathrm{P}}=0.32$ with specific velocities, respectively $Z=0.70$ and $Z=0.80$. This is greater than the maximum value of the power factor $C_{\mathrm{P}}=0.20$, obtained when testing the Savonius rotor in the hydrobasin [19].

\section{Recommendations for improving the design of the rotors of VA WT}

The efficiency of the N-Darrieus rotor is strongly affected by such design elements as the crosshead for attaching the blades to the rotor shaft. In the air flow, drag forces act on the traverse, which reduce the rotating aerodynamic moment created by blades. Such assessment was performed in [19] when testing the 6-blade two-tier N-Darrieus rotor model. The resistance forces in the flow around the crosshead were so great that they led to a two-fold decrease in the maximum value of the useful power and, consequently, the power factor $C p$, which decreased from 0.56 to 0.28 , i.e. 2 times. Thus, with a rational choice of the design of the mounting system of blades, it is possible to achieve a significant reduction in the energy loss of the rotating $\mathrm{N}$-Darrieus rotor, thereby increasing the power factor $C_{p}$, i.e. its energy efficiency.

Very important is the role of the angle of installation of the airfoil blades. Previously performed experiments by the authors showed that increasing the angle of attack of the blades from $0^{\circ}$ to $4^{\circ}$ resulted in an increase of the N-Darrieus rotor speed by an average of $17 \%$. Since the developed power of rotor machines is proportional to the cubed rotational speed, the power of the wind turbine increased for 1.6 times, and the power factor should increase for approximately the same degree. This is confirmed by experiments described in [19]. These experiments showed that with an increase in the angle of installation of the blades $\alpha$ from $0^{\circ}$ to $4^{\circ}$, the power factor $C_{p}$ increased from 0.40 to 0.61 , i.e. for 1.5 times. Thus, at the optimum angle of blades installation $\left(4^{\circ}\right)$, it is possible to increase the power factor $C_{\mathrm{P}}$ by $1.5-1.6$ times.

The power factor $C_{P}$ of the rotor N-Darrieus is also influenced by the filling factor of the rotor profile $\sigma$. This coefficient depends on the ratio between the chord length $b$ of the airfoil and the rotor diameter $D$. For example, in the experimental setup [19] with increasing $b$ from 0.03 to $0.08 \mathrm{~m}$ and at constant rotor diameter $D=0.65 \mathrm{~m}$, the filling factor $\sigma$ increased from of power factor $C_{P}$ towards higher values of specific velocity [19]. This can be explained by the fact that an increase in specific velocity leads to the suppression of formation and disruption of the dynamic flow from the blades of N-Darrieus, thereby reducing losses and increasing the energy efficiency of the VA WT by $5-18 \%$.

The use of optimal geometric ratios of parameters, such as the ratio between the height of blades and rotor diameter $\mathrm{H} / \mathrm{D}=0.77$, the relation between distance from the shaft axis to the edge of the blade $p_{k}$ to the blade radius $p / r=0.20$, the central angle of the circular arcs $\theta=135^{\circ}$ of the Savonius rotor allows one to achieve the maximum possible power factor $C_{\mathrm{P}}=0.27$, and additional use of flaps with triangular elements allows one to achieve $C_{\mathrm{P}}=0.32$. Thus, it is advisable to supply the Savonius rotor by semi-cylindrical blades with optimal geometrical dimensions with flaps in the form of triangular zigzags. The maximum possible 
value for HO WT, $C_{\mathrm{P}}=0.45$, is achieved only when the wind speed coincides with the axis of rotor. Their wind control systems reduce the actual power factor to $0.25-0.35[13,20]$. Therefore, the Savonius rotor design, proposed by the authors, with the maximum possible power factor of 0.32 , can be quite competitive with HO WT.

Thus, the authors found that the rotational speed of the original N-Darrieus and Savonius rotors, having blades with flaps, is $18 \%$ higher than the rotational speed of the N-Darrieus and Savonius rotors of the known design, and the power factors of the N-Darrieus rotors are $17 \%$ and $19 \%$, respectively, so it is advisable to use flaps with triangular elements. Optimization of geometrical parameters and improvement of the forming blades can lead to an increase in the N-Darrieus rotor to a value of 0.72 exceeding the maximum possible value for HO WT $C_{P}=0.45$. This will make VA WT competitive to HO WT and in the future will be able to significantly increase their share of the total WT production.

The proposed N-Darrieus and Savonius rotors of optimal geometrical parameters with flaps on can be widely applied for power supply of autonomous objects on land and at sea. More high-speed N-Darrieus rotors is advisable to aggregate with Savonius rotors, which have a sufficiently large starting torque. Therefore, the launch of the N-Darrieus rotor can be carried out at much lower rotational speeds. Such a combined rotor can be arranged with an electric generator, as well as with mechanical heat generator and used in the systems of electricity and heat supply of various objects: low-rise buildings, farms, tourist complexes and field objects, etc. The N-Darrieus rotors, Savonius rotors and combined flap rotors proposed by the authors can also be used to power river and sea vessels, as well as to drive propellers for small vessels.

\section{Conclusions}

1. For the first time, experimental studies have been performed to assess the effect of zigzag flaps on the operation of the N-Darrieus and Savonius rotors. It has been established that the rotor frequency of the original N-Darrieus and Savonius rotors having blades with flaps is $18 \%$ greater than that for the N-Darrieus and Savonius rotors of known design, and the power factors of the N-Darrieus rotors are greater for $17 \%$ and $19 \%$, respectively. This is explained by the fact that the zigzag flaps break large vortices that form in the aft part of the foil of blades or behind the outer generatrix of the semi-cylindrical blades during the rotation of the Savonius rotors. Thus, it is advisable to supply the VO WT blades with flaps with triangular elements of small height.

2. The resistance forces in the flow around the Darrieus rotor traverse are so great that they can lead to a decrease of power factor from 0.56 to 0.28 , i.e. for 2 times. At the optimum angle of installation of the blades $\left(4^{\circ}\right)$ and with flaps in the form of triangular zigzags, the rotor speed of the N-Darrieus increases, which will lead to an increase in power factor. Optimization of geometrical parameters and improvement of blade designs can lead to an increase in the N-Darrieus rotor power factor to a value of 0.72 higher than the maximum possible value of 0.45 for $\mathrm{HO} \mathrm{WT}$.

3. The maximum possible value for HO WT, $C_{P}=0.45$, is achieved only when the wind speed coincides with the axis of rotation of rotor. They possess sufficiently inertial wind orientation control systems reduces the actual power factor to $0.25-0.35$. Therefore, the Savonius rotor design, proposed by the authors, with the maximum possible power factor of 0.32 , can be quite competitive with HO WT.

4. N-Darrieus rotors should be aggregated with Savonius rotors, which have a sufficiently large starting torque. Therefore, the launch of the N-Darrieus rotor can be carried out at much lower rotational speeds. Such a combined rotor can be arranged with an electric generator, as well as with a mechanical heat generator and used in the systems of 
electricity and heat supply of various objects. These rotors can also be used to power river and sea vessels and to drive propellers for small vessels.

\section{References}

1. B.V. Semkin, M.I. Stalnaya, P.P. Sweet,Thermal Engineering 2, 6-7 (1996)

2. N.D. Shishkin, Efficient use of renewable energy sources for autonomous heating of various facilities (ASTU publishing house, Astrakhan, 2012)

3. A.I. Chivenkov, A.B. Loskutov, E.A. Mikhailichenko, Industrial Energy 5, 57-63 (2012)

4. N.D. Shishkin, R.A. Ilyin, Vestnik ASTU 2, 42-50 (2017)

5. V. Elistratov, R. Denisov, Proceedings of 2017 International Conference on Industrial Engineering, Applications and Manufacturing, ICIEAM 2017, 8076219 (2017)

6. V.V. Potekhin, D.N. Pantyukhov, D.V. Mikheev, EAI Endorsed Transactions on Energy Web 17(11), e5 (2017)

7. Y.A. Makarichev, A.S. Anufriev, Y.N. Ivannikov, N. Didenko, A. Gazizulina, International Conference on Information Networking 2018-January, 671-672 (2018)

8. I. Konstantinov, A. Savchenko, A. Frolov, L. Akimov, M. Holmin, MATEC Web of Conferences 53, 01064 (2016)

9. V.M. Lyakhter, Yu.B. Shpolyansky, Proc. scientific Tr. Hydroproject M.: Wind power stations 129, 113-127 (1988)

10. E.V. Solomin, Methodology of development and creation of vertical-axial wind power plants: a monograph (YuRGU Publ.House, Chelyabinsk, 2011)

11. P.G. Baklushin, K.P. Vashkevich, V.V. Samsonov, Proc. scientific Tr. Hydroproject M.: Wind power stations 129, 98-105 (1988)

12. A.K. Ershina, Sh.A. Ershin, U.K. Zhalbasbayev, Basics of the theory of the Darrieus wind turbine (Kazgos INTI, Almaty, 2001)

13. E.R. Abramovsky, Wind turbine aerodynamics: Proc. manual (DGU, Makhachkala, 1987)

14. O.M. Belotserkovsky, Numerical Modeling in Continuum Mechanics (Fizmatlit, Moscow, 1994)

15. S.M. Belotserkovsky, Wing in a non-stationary gas flow (Nauka, Moscow, 1971)

16. N.I. Volkov, Aerodynamics of orthogonal wind turbines: Textbook (VVP "Mriya-1" LTD, Sumy, 1996)

17. D.N. Gorelov, Thermophysics and Aeromechanics 17(3), 325-333 (2010)

18. D.N. Gorelov, Thermophysics and Aeromechanics 10(1), 47-51 (2003)

19. D.N. Gorelov, Aerodynamics windwheels with a vertical axis of rotation (Polygraphic Center of CAS, Omsk, 2012)

20. N.D. Shishkin, R.A. Ilyin, Vestnik ASTU 2, 42-50 (2018)

21. Schematic drawings of the wings of birds https://yandex.ru/images/search?text=schematic+drawing+bird+wing (2018)

22. M. Forando, Mod. Engi. Ser. A 10, 139-148 (1988)

23. A.A. Bubenchikov, Ye.V. Pomogalova, V.A. Zhdanova, G.A. Kovalev, Young Scientist 22.3, 14-18 (2016)

24. N.D. Shishkin, I.S. Terentyev, ASTU Bulletin, ser. Marine equipment and technology 2, 56-63 (2015) 
25. N.D. Shishkin, R.A. Ilyin ASTU Bulletin, ser. Marine equipment and technology 3, 93100 (2018)

26. T. Ogawa, H. Yoshida, Y. Yokota, Mod. Engi. Ser. A 10, 60-66 (1989) 